\title{
Performances de lapines de souche synthétique algérienne conduites en insémination artificielle : effet de la saison
}

\author{
Khedidja Boudour ${ }^{1 *}$ El Hassen Lankri ${ }^{1}$ \\ Nacira Daoudi Zerrouki ${ }^{2}$ Ahmed Aichouni ${ }^{3}$
}

\section{Mots-clés}

Oryctolagus, lapin, poids, fertilité, prolificité, mortalité, saison, Algérie

Submitted: 19 October 2019

Accepted: 19 February 2020

Published: 29 June 2020

DOI: $10.19182 /$ remvt.31880

\section{Résumé}

L'objectif de l'étude a été de caractériser les performances reproductives (fertilité, prolificité, mortalités) et pondérales des lapines de la souche synthétique algérienne "ITELV 2006 », conduites en insémination artificielle (IA) avec un rythme semi-intensif (intervalle mise bas - IA de 12 jours). Durant deux années consécutives (2017 et 2018), un suivi de la conduite de reproduction de 73 lapines, faisant l'objet de 327 IA, a été mené à I'animalerie de I'Université de Chlef (Algérie). Durant la durée de l'étude, les IA ont été réalisées à partir d'une semence récoltée chez des mâles du même type génétique et analysée localement. Les données relatives à I'IA, à la palpation, à la mise bas et au sevrage ont été enregistrées pour l'ensemble des femelles. L'effet de la saison sur les critères de poids et les performances de reproduction des lapins a été analysé. Toutes les données ont été soumises à une analyse de variance. Les femelles ont montré une bonne prolificité de 9,5 $\pm 2,4$ nés totaux dont 8,4 $\pm 2,9$ nés vivants par mise bas et 7,4 $\pm 2,6$ sevrés par sevrage, avec des taux de fertilité de 85,6 $2 \pm 35,1 \%$ à la palpation et $69,7 \pm 45,9 \%$ à la mise bas. Le poids adulte des femelles à I'IA était de $3584 \pm 391 \mathrm{~g}$. Le lapereau pesait en moyenne $63 \pm 13 \mathrm{~g}$ à la naissance et $490 \pm 81 \mathrm{~g}$ au sevrage. Un effet très significatif de la saison a été enregistré sur la fertilité et la prolificité des lapines, et sur le poids et la mortinatalité des lapereaux avec les plus faibles valeurs enregistrées en saison chaude $63 \%$ de fertilité). Cependant, les femelles sont restées productives durant cette période de grande chaleur.

- Comment citer cet article : Boudour K., Lankri El H., Zerrouki N.D., Aichouni A., 2020. Performances of Algerian-synthetic-strain rabbits managed with artificial insemination: Effect of the season. Rev. Elev. Med. Vet. Pays Trop., 73 (2): 91-98, doi: 10.19182/remvt.31880

\section{INTRODUCTION}

Un programme de recherche a été mis en place pour améliorer le potentiel génétique des lapins destinés à la production de viande en Algérie. Il a abouti à la création et la diffusion d'un nouveau génotype cunicole appelé « ITELV 2006 ». La souche est sélectionnée depuis décembre 2003 (Gacem et al., 2009) dans le but d'avoir une bonne

\footnotetext{
1. Laboratoire de bio ressources naturelles, Université Hassiba Benbouali, Chlef,
Algérie.

1. Laboratoire de bio ressources naturelles, Université Hassiba Benbouali, Chlef,
Algérie.

2. Laboratoire ressources naturelles, Université Mouloud Maameri, Tizi Ouzou, Algérie.

3. Centre universitaire El wancharissi, Tissemsilet, Algérie.

* Auteur pour la correspondance

Tél. : +213 (0)2 7727906 ; email : k.boudour@univ-chlef.dz
}

croissance et une meilleure adaptation aux conditions climatiques de l'Algérie.

Depuis sa diffusion chez les éleveurs en février 2012, cette souche synthétique a fait l'objet de nombreux travaux qui ont consisté tout d'abord à décrire ses caractéristiques. La majorité de ces travaux a porté sur une description des caractéristiques en comparaison avec les populations locales (Bolet et al., 2012 ; Zerrouki et al., 2014 ; Sid et al., 2018a). La conduite de la reproduction est la saillie naturelle, avec un rythme semi-intensif appliqué dans les élevages étatiques et privés, et se définissant par un intervalle mise bas - insémination artificielle (IA) de 11 à 12 jours. Ces études mettent en évidence des différences plus ou moins importantes sur le poids de la femelle à la saillie, à la palpation et à la mise bas, et sur la taille des portées à la naissance et au sevrage, en faveur de la souche synthétique.

Bien que la maîtrise de la technique de l'IA ait permis la mise en place d'un nouveau système de production se définissant par la conduite en 
bande, permettant d'inséminer toutes les femelles en même temps et assurant ainsi une meilleure organisation des élevages en réduisant les tâches et le temps consacrés à la reproduction (Dal Bosco et al., 2011), peu de travaux scientifiques ont utilisé ce mode de reproduction pour la souche synthétique ITELV 2006.

Pour bénéficier des progrès génétiques qui ont mené à la création de la souche synthétique et définir les meilleures conditions pour la production, la présente étude a eu pour objectif l'évaluation des performances de reproduction (fertilité et prolificité des femelles reproductrices, mortalités et poids des lapereaux à la naissance et au sevrage) d'un cheptel de lapines de la souche synthétique tout en utilisant l'IA et en analysant l'effet de la saison sur les paramètres étudiés.

\section{MATERIEL ET METHODES}

\section{Animaux}

Soixante-treize lapines, de couleur de robe très hétérogène, appartenant à la souche ITELV 2006 acquise auprès de l'Institut technique des élevages (ITELV) à la station Lamtar d'Alger, ont été utilisées. Ce génotype a été créé par IA entre des femelles de la population locale algérienne et des mâles de la souche française « INRA 2666 », sélectionnée pour sa prolificité (9 lapereaux nés vivants par mise bas et 7,5 sevrés ; Gacem et al., 2008), suite à une coopération entre l'ITELV et l'Institut national de la recherche agronomique (INRA) de Toulouse.

\section{Conditions expérimentales}

L'expérimentation s'est déroulée à l'animalerie de l'université de Chlef, pendant 12 mois. De janvier à juin 2017, 38 lapines ont été utilisées puis, de juillet à décembre 2018 et au début de cette deuxième période, 35 lapines de poids et d'âge comparable les ont remplacées en totalité. Les animaux ont été logés dans un bâtiment construit en dur, disposant de fenêtres assurant l'aération et l'éclairage naturel, et muni d'extracteurs pour la ventilation. Les cages disposées en flatdeck (surélevées et à un seul niveau) et conçues avec un grillage métallique mesuraient $60 \mathrm{~cm}$ de longueur sur $45 \mathrm{~cm}$ de largeur et $30 \mathrm{~cm}$ de hauteur. Les cages des reproductrices étaient équipées de boîtes à nid de $30 \mathrm{~cm} \mathrm{x} 45 \mathrm{~cm}$. Les lapins sevrés et les reproducteurs mâles ont été logés dans des cages d'engraissement. Les cages de maternité et d'engraissement étaient équipées d'une mangeoire à distribution manuelle, et l'abreuvement était automatique et fourni à volonté. Les animaux recevaient un aliment standard commercial sous forme de granulés, contenant $14,5 \%$ de protéines brutes et $9,5 \%$ de cellulose brute.

L'hygiène du bâtiment était assurée par le nettoyage quotidien des clapiers. Les cages de maternité étaient désinfectées après chaque sevrage. Létat de santé des mères et de leurs portées était contrôlé quotidiennement et les individus morts étaient immédiatement sortis du bâtiment d'élevage. La prophylaxie était assurée par l'administration d'un vaccin antiparasitaire (Ivomec) et d'un vaccin contre les entérotoxémies (Coglavax), ainsi que par l'addition de vitamines $C$ et choline pour prévenir les troubles digestifs lors du sevrage, et de minéraux dans l'eau de boisson afin de prévenir les problèmes liés aux carences.

\section{Conduite de la reproduction}

Les soixante-treize lapines ont été inséminées pour la première fois à un âge de quatre à cinq mois et un poids moyen de $3250 \pm 407$ g. L'intervalle mise bas - IA était de 12 jours. Au total 327 IA ont été réalisées avec des mâles de même type génétique. Au début de l'essai, leur âge variait de 7,5 à 8 mois et ils pesaient en moyenne 3745 \pm 381 g. Les éjaculats ont été analysés immédiatement après collecte. S'ils étaient de qualité et quantité suffisantes, ils étaient dilués 10 fois dans un dilueur commercial (Galap).
Les femelles étaient inséminées avec une dose de 0,3 ml de semence diluée, juste après avoir reçu $0,3 \mathrm{ml}$ de gonadolibérine (GnRH) injectée en intramusculaire pour déclencher l'ovulation. Les lapines ont été totalement renouvelées pour l'année 2018 avec des lapines du même poids et du même âge que celles de l'année précédente (2017). Durant les deux périodes de l'expérimentation, les lapines mortes ont été remplacées pour maintenir l'effectif constant.

Le diagnostic de gravidité a été fait par palpation abdominale 12 jours après l'IA. Les femelles non gravides étaient à nouveau inséminées le jour même ou au plus tard le lendemain de la palpation. A l'approche de la mise bas (à 4 ou 5 jours de la date prévue pour la mise bas), des boîtes à nid étaient placées dans la cage des lapines. Le nombre de lapereaux nés vivants et mort-nés a été dénombré après la mise bas. Les lapereaux vivants ont été pesés collectivement. Le sevrage a été réalisé à 30 jours. Les lapereaux ont été ensuite transférés dans des cages d'engraissement après dénombrement, pesée et sexage.

\section{Contrôles effectués}

Le poids de la femelle, la date de l'IA, la date de la palpation, le poids de la femelle à l'IA, le résultat de la palpation, le poids de la femelle à la palpation, la date de la mise bas, le poids de la femelle à la mise bas, le poids de la portée vivante, le nombre total de lapereaux (NT), le nombre de lapereaux nés vivants (NV), le nombre de mort-nés (MN), la date du sevrage, le poids individuel du lapereau sevré, le nombre et le sexe des lapereaux sevrés (NS) ont été enregistrés.

\section{Variables étudiées}

Nous avons étudié le taux de gravidité (nombre de femelles palpées positivement / nombre de femelles inséminées), le taux de mise bas (nombre de femelles ayant mis bas / nombre de femelles inséminées), la prolificité à la naissance (NV / nombre de mises bas), la mortinatalité ([NT-NV]/NT×100), les mortalités naissance-sevrage ([NV-NS]/ $\mathrm{NV} \times 100)$, le poids moyen des femelles à l'IA, à la palpation et à la mise bas, le poids de la portée à la naissance et au sevrage, et le poids moyen des lapereaux à la naissance et au sevrage.

\section{Effet considéré}

Nous avons considéré l'effet saison de l'IA avec trois niveaux comme définis par Zerrouki et al. (2014), divisant l'année en trois saisons de quatre mois, en relation avec les températures habituellement observées et en tenant compte du climat de la zone d'étude : la période qui précède la saison chaude $(\mathrm{AV})$ du $1^{\mathrm{er}}$ février au 31 mai, la saison chaude $(\mathrm{CH})$ du $1^{\mathrm{er}}$ juin au 30 septembre, et la période postérieure à la saison chaude (AP) du $1^{\text {er }}$ octobre au 31 janvier.

\section{Analyse statistique}

Les résultats obtenus pour l'ensemble des variables (mesurées et calculées) ont été analysés avec le logiciel XLstat2016 en faisant une analyse de variance (Anova) prenant en compte l'effet de la saison de l'IA. Dans le cas de différences significatives, les moyennes ont été comparées deux à deux par le test de Fisher.

\section{RESULTATS}

Durant 12 mois (de janvier à juin 2017, et de juillet à décembre 2018), 327 inséminations ont été réalisées chez les 73 lapines de la souche synthétique. Elles ont donné lieu à 228 mises bas, 1920 lapereaux nés vivants et 1580 lapereaux sevrés sur 213 sevrages. Les critères pondéraux des lapines et de leurs portées, les performances reproductives des femelles ainsi que l'effet de la saison ont été analysés. 


\section{Performances pondérales des lapines et de leurs portées}

Les lapines de la souche synthétique pesaient en moyenne 3584 $\pm 391 \mathrm{~g}$ à l'IA, $3707 \pm 346 \mathrm{~g}$ à la palpation, et $3630 \pm 346 \mathrm{~g}$ à la mise bas. Le poids moyen du lapereau était de $63 \pm 13 \mathrm{~g}$ à la naissance et de $490 \pm 81 \mathrm{~g}$ au sevrage (tableau I).

\section{Performances de reproduction}

Le tableau II montre les moyennes des performances de reproduction (taux de gravidité, taux de mise bas, mortalités, nombre de lapereaux nés, morts et sevrés). La fertilité des lapines estimée soit par le taux de gravidité (résultat de la palpation), soit par nombre de mises bas par rapport au nombre de femelles inséminées a été respectivement de $85,6 \pm 35,1 \%$ et de $69,7 \pm 45,9 \%$. La prolificité des lapines a été de 9,5 $\pm 2,4$ nés totaux dont $8,4 \pm 2,9$ nés vivants et 7,4 $\pm 2,6$ sevrés. Les mortalités enregistrées étaient de $13,5 \%$ à la naissance et $13,7 \%$ au nid.

\section{Effet de la saison sur les performances pondérales}

La saison a influencé significativement tous les caractères pondéraux des lapines et de leurs portées (tableau III). Les femelles inséminées avant ou après la $\mathrm{CH}$ ont été significativement plus lourdes (3651 g et $3616 \mathrm{~g} ; \mathrm{p}=0,001)$ que celles inséminées pendant la $\mathrm{CH}(3471 \mathrm{~g})$.

\section{Tableau I}

Critères pondéraux chez les lapines et les lapereaux de souche synthétique ITELV 2006 en Algérie

\begin{tabular}{llrrrrr} 
Critère & n & Moy & ET & CV & Min & Max \\
\hline PIA (g) & 327 & 3584 & 391 & 0,10 & 2490 & 4495 \\
PPI (g) & 327 & 3707 & 346 & 0,09 & 2730 & 4585 \\
PMB (g) & 228 & 3630 & 346 & 0,09 & 2735 & 4470 \\
PPV (g) & 228 & 535 & 199 & 0,37 & 43 & 905 \\
PMV (g) & 228 & 63 & 13 & 0,20 & 39 & 87 \\
PMS (g) & 213 & 490 & 81 & 0,16 & 373 & 708
\end{tabular}

ET : écart-type ; CV : coefficient de variation ; Min : minimum ; Max : maximum ; PIA : poids de la femelle à l'insémination artificielle; PPl : poids de la femelle à la palpation ; PMB : poids de la femelle à la mise bas ; PPV : poids de la portée vivante ; PMV : poids moyen du lapereau vivant ; PMS : poids moyen du lapereau sevré

\section{Tableau II}

Performances de reproduction des lapines de souche synthétique ITELV 2006 en Algérie

$\begin{array}{lcrrrrr} & \text { n } & \text { Moy } & \text { ET } & \text { CV } & \text { Min } & \text { Max } \\ \text { TG (\%) } & 327 & 85,6 & 35,1 & 0,48 & 0 & 100 \\ \text { TMB (\%) } & 327 & 69,7 & 45,9 & 0,66 & 0 & 100 \\ \text { NT } & 228 & 9,5 & 2,4 & 0,24 & 2 & 12 \\ \text { NV } & 228 & 8,4 & 2,9 & 0,35 & 0 & 12 \\ \text { MN } & 228 & 1,1 & 1,6 & 1,48 & 0 & 8 \\ \text { MNT (\%) } & 228 & 13,5 & 21,9 & 1,62 & 0 & 100 \\ \text { NS } & 213 & 7,4 & 2,6 & 0,34 & 1 & 12 \\ \text { MN-S (\%) } & 213 & 13,7 & 13,7 & 1,00 & 0 & 100\end{array}$

Moy : moyenne ; ET : écart-type ; CV : coefficient de variation ; Min : minimum ; Max : maximum; TG : taux de gravidité ; TMB : taux de mise bas ; NT : nés totaux ; NV : nés vivants ; MN : mort-nés ; MNT : mortinatalité ; NS : nombre de sevrés ; MN-S : mortalités entre la naissance et le sevrage

\section{Tableau III}

Variation des caractères pondéraux ( $\mathrm{g}$ ) des lapins de souche synthétique ITELV 2006 en fonction de la saison en Algérie

\begin{tabular}{|c|c|c|c|c|}
\hline Saison & AV & $\mathrm{CH}$ & AP & $\mathbf{P}$ \\
\hline PIA & $\begin{array}{c}3651 \mathrm{a} \pm 400 \\
(\mathrm{n}=151)\end{array}$ & $\begin{array}{c}3471 b \pm 406 \\
(n=108)\end{array}$ & $\begin{array}{c}3616 a \pm 295 \\
(n=68)\end{array}$ & 0,001 \\
\hline PPI & $\begin{array}{c}3792 \mathrm{a} \pm 334 \\
(\mathrm{n}=151)\end{array}$ & $\begin{array}{c}3593 b \pm 372 \\
(n=108)\end{array}$ & $\begin{array}{c}3701 a, b \pm 269 \\
(n=68)\end{array}$ & 0,0001 \\
\hline PMB & $\begin{array}{c}3689 a \pm 348 \\
(n=114)\end{array}$ & $\begin{array}{c}3529 b \pm 351 \\
(n=68)\end{array}$ & $\begin{array}{c}3641 a, b \pm 294 \\
(n=46)\end{array}$ & 0,01 \\
\hline PPV & $\begin{array}{c}658 a \pm 123 \\
(n=114)\end{array}$ & $\begin{array}{c}365 b \pm 196 \\
(n=68)\end{array}$ & $\begin{array}{c}480 c \pm 136 \\
(n=46)\end{array}$ & 0,0001 \\
\hline PMV & $\begin{array}{l}68 a \pm 7,5 \\
(n=114)\end{array}$ & $\begin{array}{c}55 b \pm 15 \\
(n=68)\end{array}$ & $\begin{array}{c}60 c \pm 13,2 \\
(n=46)\end{array}$ & 0,0001 \\
\hline PMS & $\begin{array}{c}506 a \pm 83 \\
(n=103)\end{array}$ & $\begin{array}{c}485 a, b \pm 89 \\
(n=64)\end{array}$ & $\begin{array}{c}462 b \pm 49 \\
(n=46)\end{array}$ & 0,007 \\
\hline
\end{tabular}

$\mathrm{AV}$ : précède la saison chaude ( 1 février - 31 mai) $\mathrm{CH}$ : saison chaude ( 1 juin -30 sept.) ; AP : suit la saison chaude (1 oct. - 31 janv.) ; PIA : poids de la femelle à

l'insémination artificielle ; PPl : poids de la femelle à la palpation; $\mathrm{PMB}:$ poids de la femelle à la mise bas ; PPV : poids de la portée vivante ; PMV : poids moyen du lapereau vivant ; PMS : poids moyen du lapereau sevré

a,b,c Sur une même ligne les valeurs sans lettre commune sont différentes au seuil de $5 \%$.

L'influence des fortes températures estivales (juin à septembre) a également été observée sur le poids de la lapine à la palpation et à la mise bas. Les plus faibles poids ont été obtenus en $\mathrm{CH}$ alors que les lapines étaient plus lourdes pendant la période précédant (AV) la $\mathrm{CH}(3593 \mathrm{~g}$ contre $3792 \mathrm{~g} ; \mathrm{p}=0,0001$, pour le poids à la palpation; et $3529 \mathrm{~g}$ contre $3689 \mathrm{~g}, \mathrm{p}=0,01$ pour le poids à la mise bas).

La saison a influencé significativement $(\mathrm{p}<0,0001)$ le poids moyen des portées à la naissance. Elles ont été plus lourdes avant les fortes chaleurs, c'est-à-dire pendant la période frâiche ( $658 \mathrm{~g}$ vs $365 \mathrm{~g}$ pour $\mathrm{CH}$, et $480 \mathrm{~g}$ pour AP) (tableau III). Les mises bas de la $\mathrm{CH}$ donnent des lapereaux moins lourds que ceux des AP et AV (respectivement $55 \mathrm{~g}$ vs $60 \mathrm{~g}$ vs $68 \mathrm{~g} ; \mathrm{p}=0,0001)$. Le poids moyen des lapereaux au sevrage a été fortement influencé par la saison $(\mathrm{p}=0,007)$. Les lapereaux sevrés avant la $\mathrm{CH}$ ont été plus lourds $(506 \mathrm{~g})$ que ceux sevrés après la $\mathrm{CH}(462 \mathrm{~g})$ ou pendant la $\mathrm{CH}(485 \mathrm{~g})$.

\section{Effet de la saison sur les performances de reproduction}

Les contrastes de poids enregistrés à travers les variations saisonnières ont été accompagnés de variations des performances de reproduction. En général, la période $\mathrm{AV}$ (saison fraîche de février à mai), pendant l'allongement de la photopériode naturelle, a permis des performances optimales (tableaux IV et V).

\section{Effet de la saison sur la fertilité et la mortinatalité}

Le taux de gravidité a varié significativement $(\mathrm{p}=0,017)$ en fonction de la saison. La valeur la moins élevée a été obtenue pendant la période succédant la $\mathrm{CH}$ (75\%), alors que le taux le plus important a été enregistré pendant la saison précédant la $\mathrm{CH}(89,4 \%)$, conduisant à un résultat intermédiaire (tableau IV). Les taux de mortalités à la naissance n'ont pas différé significativement entre la période avant la $\mathrm{CH}(7,5 \%)$ et après $(9,4 \%)$. En revanche, c'est au cours de la $\mathrm{CH}$ que le taux de mortinatalité a été le plus élevé $(26,2 \%$; $\mathrm{p}=0,0001)$. 


\section{Tableau IV}

Variation de la fertilité des lapines de souche synthétique ITELV 2006 en fonction de la saison en Algérie

$\begin{array}{lcccc}\text { Saison } & \text { AV } & \text { CH } & \text { AP } & \text { P } \\ \text { TG } & \begin{array}{c}89,4 a \pm 30,8 \\ (n=151)\end{array} & \begin{array}{c}87 a, b \pm 33,6 \\ (n=108)\end{array} & \begin{array}{c}75 b \pm 43,3 \\ (n=68)\end{array} & 0,017 \\ \text { TMB } & \begin{array}{c}75,5 a \pm 43 \\ (n=151)\end{array} & \begin{array}{c}63 b \pm 48,3 \\ (n=108)\end{array} & \begin{array}{c}67,6 a, b \pm 43,3 \\ (n=68)\end{array} & 0,031 \\ \text { MNT } & 7,5 a \pm 14,2 & 26,2 b \pm 31 & 9,4 a \pm 11 & 0,0001 \\ & (n=114) & (n=68) & (n=46) & \end{array}$

$\mathrm{AV}$ : précède la saison chaude, ( 1 février -31 mai) $\mathrm{CH}$ : saison chaude (1 juin - 30 sept.) ; AP : suit la saison chaude (1 oct. - 31 janv.) ; TG : taux de gravidité ; TMB : taux de mise bas ; MNT : mortinatalité

${ }^{\mathrm{a}, \mathrm{b}}$ Sur une même ligne les valeurs sans lettre commune sont différentes au seuil de $5 \%$.

\section{Tableau V}

Variation de la prolificité des lapines de souche synthétique ITELV 2006 en fonction de la saison en Algérie

\begin{tabular}{lcccc} 
Saison & AV & $\mathbf{C H}$ & AP & $\mathbf{P}$ \\
\hline NT & $10.5 a \pm 1,5$ & $\begin{array}{c}8.1 b \pm 2,8 \\
(n=68)\end{array}$ & $\begin{array}{c}9 b \pm 2,1 \\
(n=46)\end{array}$ & 0,0001 \\
& $(n=114)$ & $(n=1,5$ & \\
NV & $9,8 a \pm 1,9$ & $6,4 b \pm 3,5$ & $8,1 c \pm 2,2$ & 0,0001 \\
& $(n=114)$ & $(n=68)$ & $(n=46)$ & \\
MN & $0,7 a \pm 1,4$ & $1,8 b \pm 1,9$ & $0,8 a \pm 0,9$ & 0,0001 \\
& $(n=114)$ & $(n=68)$ & $(n=46)$ & \\
NS & $8,2 a \pm 1,9$ & $6,4 b \pm 3,2$ & $7,1 b \pm 2,5$ & 0,0001 \\
& $(n=103)$ & $(n=64)$ & $(n=46)$ &
\end{tabular}

$\mathrm{AV}$ : précède la saison chaude, (1 février -31 mai) ; $\mathrm{CH}$ : saison chaude ( 1 juin 30 sept.) ; AP : suit la saison chaude (1 oct. -31 janv.) ; NT : nés totaux ; NV : nés vivants ; MN : mort-nés ; MNT : mortinatalité ; NS : nombre de sevrés

a,b,c Sur une même ligne les valeurs sans lettre commune sont différentes au seuil de $5 \%$.

\section{Effet de la saison sur la prolificité}

L'effet positif de la période fraîche a été observé sur la prolificité des lapines $(10,5 \mathrm{NT}, 9,8 \mathrm{NV}$ et 8,2 NS; $\mathrm{p}=0.0001)$. A l'opposé, le nombre de lapereaux vivants a été le plus faible $(6,4)$ et le nombre de mort-nés le plus élevé $(1,8)$ pendant la $\mathrm{CH}$. L'effet négatif de la $\mathrm{CH}$ s'est répercuté significativement $(\mathrm{p}=0,0001)$ sur le nombre de lapereaux sevrés : les lapines inséminées à la $\mathrm{CH}$ ont sevré moins de lapereaux que celles inséminées avant (-1,8 lapereau) ou après $(-0,7$ lapereau) la CH. Cependant, le nombre de NT et de NS n'a pas varié significativement entre la $\mathrm{CH}$ et la saison qui lui a succédé (8,1 et 9 pour les NT, et 6,4 contre 7,1 pour les NS).

\section{DISCUSSION}

\section{Performances pondérales des lapines et de leurs portées}

Sid et al. (2018a) relèvent des poids moyens de $3260 \pm 450 \mathrm{~g}$ à la saillie, $3370 \pm 430 \mathrm{~g}$ à la palpation et $3190 \pm 360 \mathrm{~g}$ à la mise bas pour une durée d'essai de 18 mois. Lors des premiers travaux sur la sélection et l'homogénéisation de cette souche synthétique à la station de Baba Ali (Alger), Gacem et al. (2009) enregistrent un poids moyen à la saillie similaire à celui enregistré chez nos lapines (3633 g). Bolet et al. (2012) obtiennent un poids moyen à la mise bas moins élevé
(3436 g), Chibah (2016) un poids beaucoup moins élevé (3163 g), mais Zerrouki et al. (2014) un poids (3599 g) comparable à celui enregistré chez nos lapines. Comparés aux résultats de recherches menées sur la même souche synthétique dans d'autres régions d'Algérie, ceux de notre étude indiquent, en général, une supériorité du poids des lapines, reflet de leur bon état général.

Le poids à la mise bas obtenu dans cette étude a été supérieur à celui obtenu après quatre générations d'homogénéisation et de sélection de la souche synthétique ITELV 2006 (3436 g ; Bolet et al., 2012). Il a également été supérieur à ceux obtenus par Sid et al. (2018b) et Zerrouki et al. (2014) (respectivement $3148 \mathrm{~g}$ et $3599 \mathrm{~g}$ ). Sid et al. (2014 ; 2018b) trouvent des poids à la mise bas inférieurs à ceux obtenus au cours de cette étude (3083 g et 3118,5 g) pour des lapines de la population blanche.

Le poids moyen du lapereau à la naissance $(63 \mathrm{~g})$ a été plus élevé que ceux signalés par Zerrouki et al. (2007) et Abdelli et al. (2014) chez la population locale qui sont respectivement de $51 \mathrm{~g}$ et $54 \mathrm{~g}$, et que ceux rapportés chez les lapereaux de la même souche synthétique par Gacem et al. (2009), Zerrouki et al. (2014), et Chibah et Zerrouki (2015) qui mentionnent un poids moyen du lapereau à la naissance entre $52 \mathrm{~g}$ et $54 \mathrm{~g}$. Golimytis et al. (2016) rapportent des poids moyens supérieurs chez les lapereaux nés vivants de la souche Hyla (71,5 g). Par ailleurs, Chibah et Zerrouki (2015) enregistrent chez les lapins de même souche un poids moyen du lapereau, sevré à 35 jours, plus élevé que celui enregistré dans notre étude (667 g vs $490 \mathrm{~g}$ ). Sur un autre échantillon de cette souche et de même origine (station Lamtar), Zerrouki et al. (2014) rapportent un poids moyen au sevrage légèrement supérieur (564 g) avec cependant un sevrage plus tardif (35 j vs $30 \mathrm{j}$ dans notre étude). Cette différence de poids peut être expliquée par l'allongement de la période présevrage et possiblement par un meilleur état corporel de nos femelles. Nos résultats se situent dans les normes de la production cunicole rapportées par Lebas (1996) (500 g pour le poids moyen au sevrage à 26-30 jours).

Cette souche synthétique peut être ainsi classée dans un format moyen (Sid et al., 2018a), tant au niveau du poids des lapines que de celui des lapereaux sevrés. Cependant, l'aliment granulé utilisé dans cette étude ne semble pas totalement satisfaire les besoins nutritionnels des reproductrices. Il renferme seulement $14,5 \%$ de protéines et 9,5\% de cellulose brute, quantités inférieures aux recommandations pour l'alimentation du lapin. Lebas et al. (1996) recommande une teneur de $18 \%$ en protéines et de $12 \%$ de cellulose brute. Lankri et al. (2019) montrent qu'une augmentation du taux protéique du même aliment utilisé dans la présente étude permet d'améliorer considérablement les performances de reproduction, en particulier l'ardeur sexuelle chez les mâles du même génotype.

\section{Performances de reproduction des lapines}

\section{Fertilité}

Le taux de mise bas de $69,7 \%$ relevé dans notre étude a été supérieur à ceux de $51 \%$ rapportés par Gacem et al. (2009) et 61,9\% obtenus par Zerrouki et al. (2014), respectivement dans les stations de Baba Ali et Lamtar, chez des lapines de même génotype, conduites en saillie naturelle et dans des conditions d'élevage différentes. Sid et al. (2018b) enregistrent un taux de fertilité de 66,6\%. Ces différences de fertilité peuvent être dues au mode de reproduction pratiqué dans notre étude (IA). En effet, Amroun et al. (2018) confirment que l'adoption de cette technique comme nouveau mode de reproduction avec traitement hormonal effectué par l'éleveur (injection de $\mathrm{GnRh}$ ), améliore relativement les performances des lapines à travers l'augmentation du taux de fertilité qui passe de 76,7 et $78 \%$ en saillie naturelle à 90,1 et 96,4\% en IA. En saillie naturelle seules les lapines qui sont en ostrus acceptent l'accouplement et sont saillies. 
Les différences de fertilité peuvent être également liées aux types génétiques. Bolet et al. (2004) montrent que la fertilité est en rapport avec le type génétique notamment avec le format de la souche ou de la population. Daboussi (2014) rapporte un taux de fertilité nettement inférieur chez les lapines de population locale tunisienne conduites en saillie naturelle (60\%). Sid et al. (2018b) obtiennent un taux de $60,4 \%$ chez les femelles de la population blanche. Moula et Yakhlef (2007) enregistrent un taux de fertilité supérieur (87\%) chez des lapines de population locale algérienne.

\section{Prolificité}

Dans nos conditions d'élevage, la prolificité des lapines a été supérieure à celle enregistrée chez la même souche par Sid et al. (2018a) en termes de lapereaux nés vivants $(8,4$ vs 8,0$)$ et sevrés $(7,4$ vs 6,1$)$. En revanche, Gacem et al. (2009) rapportent des résultats comparables aux nôtres avec 7,1 $\pm 0,1$ sevrés par femelle ayant mis bas pour un nombre de nés totaux de 9,5 et 8,7 nés vivants. Avec la même souche, Zerrouki et al. (2014) obtiennent des tailles de portée à la naissance de 9,6 nés totaux, 8,6 nés vivants et 6,6 sevrés. Le nombre de sevrés enregistré dans notre étude et sur la même souche était plus élevé, bien que les mortalités durant la phase naissance-sevrage aient été élevées $(13,7 \%)$ en raison, probablement, des conditions d'élevage et de la qualité des aliments. Le nombre de sevrés dépend ainsi de la viabilité des jeunes sous la mère et des qualités maternelles (Chibah et Zerrouki, 2015).

\section{Mortalités}

La mortinatalité des lapereaux pouvait être liée au comportement maternel des lapines qui ne préparaient pas correctement leur nid ou mettaient bas hors du nid ce qui était à l'origine de la perte d'une grande partie de certaines portées. Néanmoins, notre résultat (13,5\%) demeure inférieur à celui de $24 \%$ relevé par Kennou et Lebas (1990) chez les lapines de population locale tunisienne, et à celui de 17,5\% de Moumen (2017) chez des lapines de population locale aurèsienne en Algérie. Cependant Sid et al. (2018b) notent des taux de mortinatalité de 9,8\% et $18,1 \%$ respectivement chez des lapines de la souche synthétique et de la population blanche.

Le taux de mortalités entre la naissance et le sevrage a été moins élevé que ceux rapportés par Sid et al. (2018a), et Moumen (2017), respectivement de $13,7 \%$ vs $26,1 \%$ et $19,4 \%$. Ce pourrait être lié au poids des lapereaux à la naissance, relativement plus élevé (63 g vs $53 \mathrm{~g}$ ) dans notre étude. En effet, les mortalités des lapereaux entre la naissance et le sevrage dépendent en grande partie de leur poids à la naissance et de la taille de leur portée (McNitt et Lukefahr, 1990). De même, Gacem et al. (2009) observent de fortes mortalités naissancesevrage (17\% vs 13,7 \% dans notre étude) pour la souche synthétique.

\section{Effet de la saison sur les performances pondérales et les portées}

Les résultats obtenus en relation avec les variations saisonnières et l'effet dépressif de la $\mathrm{CH}$ sur les caractères pondéraux des lapines et de leurs lapereaux confirment ceux obtenus par Zerrouki et al. (2005) chez la population locale, et Gacem et al. (2009), Zerrouki et al. (2014), et Sid et al. (2018a) chez des lapines de même type génétique. La saison estivale affaiblit le poids des lapereaux à la naissance.

En conditions d'élevage tropicales et en saillie naturelle, Deprès et al. (1994) rapportent qu'un complément de six heures d'éclairement augmente la taille moyenne des portées au sevrage (6,6 vs 5,5 sevrés pour le lot témoin) mais n'améliore pas le poids des lapereaux au sevrage. L'expression des capacités réelles de production des lapines et de leurs portées en termes de poids nécessite une meilleure mâ̂trise des conditions de production, notamment l'amélioration de la qualité de l'aliment et des conditions d'élevage en $\mathrm{CH}$.

\section{Effet de la saison sur les performances de reproduction}

\section{Effet sur la fertilité et la mortinatalité}

A des températures élevées, les lapins diminuent leur consommation alimentaire (Marai et al., 2007). Les femelles gravides réduisent leur ingestion pour diminuer l'énergie produite par le métabolisme et maintenir une température corporelle stable (Villagrá et al., 2004). Ceci entraîne une mauvaise couverture des besoins énergétiques liés à la gravidité, diminuant la croissance fotale, augmentant les risques de mortalités à la naissance et la diminution du poids du lapereau né (García et al. 2019). L'effet dépressif de la CH sur les taux de fertilité peut donc être dû à une perte de poids suite à une diminution de l'ingestion d'aliment (Piles et al., 2012).

Saleil et al. (1998) rapportent qu'en CH les conditions climatiques du jour au moment de la saillie ont une influence marquée sur la fertilité des lapines élevées en plein air. Ceci se manifeste par une diminution de l'intensité d'ovulation et une augmentation des mortalités embryonnaires (Marai et al., 1996).

Durant la période AP succédant à la $\mathrm{CH}$ et correspondant en grande partie à la saison automnale, les lapines ont eu un taux de fertilité relativement faible $(67,6 \%)$. Cela pouvait être dû à une durée d'éclairement moindre en automne. Theau-Clément et Mercier (2004) montrent que l'éclairement, de par son intensité et sa durée, influence le taux d'ovulation qui se retrouve déprimé lorsque les jours décroissent en automne. Par ailleurs, une complémentation de cinq heures d'éclairage artificiel en fin de journée permet d'atténuer cet effet dépressif de l'éclairement et d'améliorer les résultats de la saillie en automne chez les lapines de la population locale tunisienne (Kennou, 1990). Un programme lumineux divisant les 24 heures en deux sous-unités de « huit heures d'éclairement + quatre heures d'obscurité » permet d'améliorer le taux de fertilité des lapines de $15 \%$ (de 67,6\% à 82,6\%) et de réduire l'intervalle entre mise bas et saillie fécondante de cinq jours (de 24,2 à 19,2 jours) (Arveux et Troislouche, 1994).

Les taux élevés de mortinatalité enregistrés pendant la $\mathrm{CH}$ pouvaient être dus au fait que la chaleur affecte la qualité des ovocytes et le nombre d'embryons viables, ce qui peut provoquer des avortements et une augmentation du nombre de lapins mort-nés (Askar et Ismail, 2012). Ainsi, les températures élevées affectent la formation et la fonction des gamètes mâles et femelles, le développement embryonnaire et la croissance fœtale chez les mammifères en reproduction (Hansen, 2009). L'effet positif de la saison printanière, correspondant en grande partie à la période $\mathrm{AV}$ dans notre étude, est rapporté par Kumar et al. (2013). Ils confirment que le printemps est la saison la plus favorable à une reproduction efficace des lapines de chair dans des conditions climatiques subtempérées de l'Inde.

\section{Effet de la saison sur la prolificité}

Goby et Rochon (1994) soulignent que les conditions climatiques printanières, en particulier la température et la photopériode, et leur évolution au cours de la saison, favoriseraient la prolificité chez la lapine. Les meilleures performances de reproduction, particulièrement la taille de la portée sevrée, sont exprimées au cours de cette saison par des lapines de différentes races (Kumar et al., 2013). De même, nos résultats corroborent ceux de Zerrouki et al. (2005) qui notent, pour des femelles nullipares, les plus faibles valeurs de prolificité de l'année en CH. Jaouzi et al. (2006) au Maroc rapportent que la CH est la plus défavorable à la reproduction, ce qui nécessiterait parfois un arrêt de la mise en reproduction des animaux pendant cette période. Comme le suggère Askar et Ismail (2012), la diminution du nombre de nés totaux et de nés vivants pourrait être liée à l'effet négatif de la 
chaleur sur la production et la qualité des follicules ovariens matures, diminuant ainsi le nombre et le développement d'embryons viables.

Le nombre faible de lapereaux sevrés enregistré en $\mathrm{CH}$ est lié à la diminution de la production laitière, elle-même due à la réduction de la consommation d'aliments sous l'effet des températures élevées (Zerrouki et al., 2005 ; Szendrő et al., 2012). Chibah et al. (2014), Chibah (2016), Amroun et al. (2018), et Zerrouki et al. (2014) montrent que l'effet dépressif de la $\mathrm{CH}$ sur le nombre de lapereaux sevrés est lié aux fortes mortalités durant la phase d'allaitement. Amroun et al. (2018) mettent en évidence un effet très significatif $(\mathrm{p}<0,0001)$ de la saison sur les mortalités des lapereaux du même génotype sous la mère en relation avec la production laitière (quantité et qualité), et confirment la variabilité de la prolificité et de la production laitière en fonction de la saison. Les lapines produisent des quantités de lait plus importantes et plus riches en hiver et au printemps que celles mesurées en été et à l'automne, rejoignant ainsi les plus forts taux de mortalités des lapereaux (Amroun et al., 2018). Ces auteurs rapportent aussi un nombre de lapereaux sevrés très faible en période estivale, avec un écart de $11,8 \%$ entre les saisons estivale et hivernale, lié à une différence de production laitière de $22 \%$ (pour la souche synthétique) et de $25 \%$ (pour la population blanche) de plus en hiver qu'en été. Selon García et al. (2019), la chaleur affecte négativement la production de lait pendant les trois premières semaines de lactation. L'animal utilise l'énergie pour maintenir sa température corporelle constante et la quantité d'éléments nutritifs nécessaires à la production de lait diminue (Askar et Ismail, 2012). Ce déficit de production laitière a pour conséquence la diminution de la croissance postnatale, du gain de poids et des chances de survie des lapereaux (Marco-Jiménez et al., 2017).

\section{- CONCLUSION}

Notre étude a contribué à caractériser les performances de reproduction de la lapine de souche synthétique ITELV 2006 conduite en insémination artificielle. Les résultats suggèrent la supériorité de la souche synthétique par rapport aux populations locales. Suivies sur période de 12 mois, les lapines ont enregistré des tailles de portées moyennes relativement élevées comparées à celles enregistrées chez les deux types locaux (populations blanche et locale). Ses performances sont très intéressantes en termes de nés vivants et de nombre de sevrés $(8,4$ et 7,4), malgré des taux de mortalités à la naissance et entre la naissance et le sevrage relativement élevés (13\%).

Les résultats obtenus sur l'introduction de l'insémination artificielle chez ces lapines ont montré l'intérêt majeur que représente son développement dans les élevages algériens. Les lapines de la souche synthétique ont préservé, dans la majorité des cas, ou même dépassé dans d'autres, les valeurs obtenues antérieurement. Elles ont enregistré de meilleures fertilités que celles rapportées par d'autres auteurs sur la même souche, conduite en saillie naturelle (taux de gravidité et de mise bas dépassant les $65 \%$ ).

La saison peut déprimer les performances de reproduction ainsi que les critères pondéraux des lapines et des lapereaux dans une région différente (Chlef, à l'ouest de l'Algérie) en raison d'un climat semiaride, plus chaud que celui des études antérieures sur cette souche (Tizi Ouzou, au centre de l'Algérie). Notre étude a mis en évidence un effet favorable du printemps (février à mai) sur la reproduction et un effet dépressif de la saison chaude, qui est resté un facteur limitant de la productivité des lapines dans les conditions d'une longue période de hautes températures. Cela n'a pas arrêté la production des lapines mais les performances ont été plus faibles que pendant les deux autres saisons en raison de meilleures conditions d'ambiance, surtout de température. Ce travail peut être poursuivi sur une plus longue période afin de mettre en évidence l'effet de cette saison combiné avec l'année sur les performances de reproduction et de production des lapines de ce type génétique.

Une étude de l'effet du mâle peut contribuer, en plus de la réceptivité sexuelle des lapines au moment de l'insémination, à améliorer l'expression des performances de reproduction des lapines. Un aspect important est la détermination des conditions d'utilisation du mâle, afin d'obtenir une quantité optimale et de bonne qualité de sperme et donc de semence. Enfin, cette étude permet de confirmer l'intérêt de la diffusion de cette souche dans les élevages pour permettre une meilleure production et assurer le développement de la cuniculture en Algérie, en raison de l'adaptation de cette souche aux conditions réelles d'élevage.

\section{Remerciements}

Les auteurs expriment leurs remerciements à la direction générale de la recherche scientifique et du développement technologique (DGRSDT) pour le financement de cette recherche.

\section{Déclaration des contributions des auteurs}

$\mathrm{KB}, \mathrm{AA}, \mathrm{NDZ}$ et EHL ont participé à la conception et à la planification de l'étude, $\mathrm{EHL}$ et $\mathrm{KB}$ ont recueilli les données ; $\mathrm{KB}$ a rédigé la première version du manuscrit, NDZ et EHL ont effectué les analyses statistiques ; NDZ a révisé le manuscrit.

\section{Déclaration de conflits d'intérêts}

L'étude a été réalisée sans aucun conflit d'intérêts.

\section{REFERENCES}

Abdelli-Larbi O., Mazouzi-Hadid F., Berchiche M., Bolet G., Garreau H., Lebas F., 2014. Pre-weaning growth performance of kits of a local Algerian rabbit population: Influence of dam coat color, parity and kindling season. World Rabbit Sci., 22: 231-240, doi: 10.4995/wrs.2014.1493

Amroun T.T., Zerrouki-Daoudi N., Charlier M., 2018. Mortalité des lapereaux sous la mère: effets de la saison de mise bas et de la production laitière des lapines de la population blanche et de la souche synthétique. Livest. Res. Rural Dev., 30, 14

Arveux P., Troislouche G., 1994. Influence d'un programme lumineux discontinu sur la reproduction des lapins. In: $6^{\text {es }}$ Journées de la recherche cunicole, La Rochelle, France, 6-7 déc. 1994

AskarA., Ismail E., 2012. Impact de l'exposition au stress thermique sur certaines caractéristiques de reproduction et physiologiques du lapin. J. Egypt. Anim. Product., 49 (2) : 151-159

Bolet G., Brun J.M., Lechevestrier S., Lopez M., Boucher S., 2004. Evaluation of the reproductive performance of eight rabbit breeds on experimental farms. Anim. Res., 53: 59-65, doi: 10.1051/animres:2003043

Bolet G., Zerrouki N., Gacem M., Brun J.M., Lebas F., 2012. Genetic parameters and trends for litter and growth traits in a synthetic line of rabbits created in Algeria. In: 10th World Rabbit Congr., Sharm El Sheikh, Egypt, 3-6 Sept. 2012, 195-199

Chibah K., 2016. Evaluation de la production laitière de la lapine et de la croissance du lapereau sous la mère de population blanche et de souche synthétique. Thèse Doct., Université Moloud Maamri, Tizi Ouzou, Algérie, $162 \mathrm{p}$.

Chibah-Ait Bouziad K., Zerrouki-Daoudi N., 2015. Effets de la taille de portée à la naissance et du nombre de lapereaux allaités sur les aptitudes laitières des lapines de deux génotypes et sur la croissance des lapereaux avant sevrage. Livest. Res. Rural Dev., 27, 224

Chibah-Ait Bouziad K., Zerrouki-Daoudi N., Amroun-Laga T., Lebas F., 2014. Effet de la taille de portée née ou allaitée sur la production laitière de lapines de deux types génétiques élevés dans des conditions d'élevage rationnelles. In : $7^{\text {es }}$ Journées de Recherche sur les productions animales, Tizi-Ouzou, Algérie, 10-11 nov. 2014

Daboussi I., 2014. Evaluation des performances génétiques des lapins reproducteurs en Tunisie. In : Sémin. Int. Elevage et faune sauvage en milieux arides et désertiques, Djerba, Tunisie, 16-18 déc. 2014, 43-44 
Dal Bosco A., Rebollar P.G., Boiti C., Zerani M., Castellini C., 2011. Ovulation induction in rabbit does: Current knowledge and perspectives. Anim. Reprod. Sci., 129 (3-4): 106-117, doi: 10.1016/j.anireprosci.2011.11.007

Deprès F., Theau-Clément M., Lorvelec O., 1994. Productivité des lapines élevées en Guadeloupe: Influence du type génétique, de I'allongement de la durée d'éclairement, de la saison et du stade physiologique. In: Journées de la recherche cunicole, La Rochelle, France, 6-7 déc. 1994, $10 \mathrm{p}$

Gacem M., Zerrouki N., Lebas F., Bolet G., 2008. Strategy for developing rabbit meat production in Algeria: creation and selection of a synthetic strain. In: $9^{\text {th }}$ World Rabbit Congr., Verona, Italy, 10-13 June 2008, 85-89

Gacem M., Zerrouki N., Lebas F., Bolet G., 2009. Comparaison des performances de production d'une souche synthétique avec les deux populations disponibles en Algérie. In: 13 ${ }^{\text {es }}$ Journées de la recherche cunicole, Le Mans, France, $17-18$ nov. 2009, 15-18

García Hernández Y., Ponce de León Senti R.E., Ledesma Rodriguez A., Rodriguez Calvo Y., Garcia Quiñones D., 2019. Influencia del nivel de calor en rasgos de prolificidad en conejos en Cuba. Livest. Res. Rural Dev., 31 (1), 4

Goby J.P., Rochon J.J., 1994. Etude comparative des résultats techniques obtenus entre une maternité en système clos et une maternité plein air dans le sud de la France (Roussillon). In: $6^{\text {es }}$ Journées de recherche cunicole, La Rochelle, France, 6-7 déc. 1994, 467-472

Golimytis M., Skoupa E.P., Konga A., Symeon G.K., Charismiadou M.A., Deligeorgis S.G., 2016. Influence of gestation maternal feed restriction on growth performance and meat quality of rabbit offsprings. Animal, 10 (1): 157-162, doi: 10.1017/S1751731115001871

Hansen P.J., 2009. Effects of heat stress on mammalian reproduction. Phil. Trans. R. Soc. B., 364: 3341-3350, doi: 10.1098/rstb.2009.0131

Jaouzi T., Barkok A., El Maharzi L., Bouzekraoui A., Archa B., 2006. Etude sur les systèmes de production cunicole au Maroc. Cunicult. Mag., 33: 99-110

Kennou S., Lebas F., 1990. Résultats de reproduction des lapines locales Tunisiennes élevées en colonies au sol. Option Méditerr., Sé. A, 8 : 93-96

Kumar D., Risam K.S., Bhatt R.S., Singh U., 2013. Reproductive performance of different breeds of broiler rabbits under sub-temperate climatic conditions. World Rabbit Sci., 21: 169-173, doi: 10.4995/wrs.2013.1196

Lankri E., Boudour K., Aichouni A., Rechachou F., 2019. Effet du niveau d'alimentation et du taux protéique de la ration sur la libido et les caractéristiques de la semence du lapin de la souche ITELV 2006. In: $18^{\text {es }}$ Journées de la recherche cunicole, Nantes, France, 27-28 mai 2019, 86-90

Lebas F., Coudert P., De Rochambeau H., Thébeault R., 1996. Le lapin, élevage et pathologie. Rome, Italie, FAO, 217 p.

Marai I.F.M., Ayyat M.S., Gaber H.A., Abdel-Monem U.M., 1996. Effect of heats stress and its amelioration on reproduction performance of New-Zealand White adult female and male rabbits, under Egyptian conditions. World Rabbit Sci., 2: 197-202

Marai I.F.M., Haeeb A.A.M., Gad A.E., 2007. Fonctions biologiques chez des jeunes lapins gravides affectés par le stress thermique et le régime d'éclairage dans des conditions subtropicales en egypte. groécosys. Trop. Subtrop., 7: 165-176
Marco-Jiménez F., García-Diego F.J., Vicente J.S., 2017. Effet de l'exposition pendant la gestation et l'allaitement au stress thermique sur la performance du lapin. World Rabbit Sci., 25: 17-25, doi: 10.4995/wrs.2017.5728

McNitt J.L., Lukefahr S.D., 1990. Effect of breed, parity, day of lactation and number of kits on milk production of rabbit. J. Anim. Sci. 68: 1505-1512, doi: 10.2527/1990.6861505x

Moula F., Yakhlef H., 2007. Evaluation des performances de reproduction d'une population locale de lapins en Algérie. In: $12^{\text {es }}$ Journées de la recherche cunicole, Le Mans, France. 27- 28 nov. 2007, 45-48

Moumen S., 2017. Influence du rythme de reproduction sur les performances de reproduction et de production des lapines de population locale : étude de la relation entre l'état nutritionnel de la femelle et sa fécondité. Thèse Doct., Université Batna, Algérie

Piles M., Bakr M.H., Tusell L., Blas E., Sanchez J.P., Ramon J., Rafael O., 2012. The effect of high environmental temperature on doe performance during lactation. In: Proc. 10th World Rabbit Congr., Sharm El- Sheikh, Egypt, 3-6 Sept. 2012, 1009-1013

Saleil G., Goby J.P., Richard F., Bochec V., 1998. Influence des conditions climatiques sur la reproduction du lapin élevé en plein-air. In : $7^{\text {es }}$ Journées de la recherche cunicole, Lyon, France, 13-14 mai 1998

Sid S., Benyoucef M.T., Mefti Korteby H., Boudjenah H., 2018a. Performances de reproduction des lapines de souche synthétique et de population blanche en Algérie. Livest. Res. Rural Dev. 30 (7), 120

Sid S., Benyoucef M.T., Mefti Korteby H., Boudjenah H., 2018b. Variation de la prolificité des lapines locales en fonction du génotype (souche synthétique et la population blanche). Rev. Agro. Bio., 8 (2) : 1001-1008

Sid S., Benyoucef M., Mefty Korteby H., Boudjenah A., Toui H., 2014. Etude comparative sur les critères de la prolificité chez les lapins des deux populations locales. In : Sémin. national de la biodiversité faunistique, Alger, Algérie, 7-9 déc. 2014

SzendrőZs., Szendrő K., Dalle Zotte A., 2012. Management of reproduction on small, medium and large rabbit farms: A review. Asian-Aust. J. Anim. Sci. 25 (5): 738-748, doi: 10.5713/ajas.2012.12015

Theau-Clément M., Mercier P., 2004. Influence of lighting programs on the productivity of rabbit does of two genetic types. In: 8th World Rabbit Congr., Puebla, Mexico, 7-10 Sept. 2004, 358-363

Villagrá A., Blanes V., Torres A., 2004. Physiologie environnementale et bioclimatique du lapin. Bull. Elev., 13-16

Zerrouki N., Hannach R., Lebas F., Saoudi A., 2007. Productivité de lapines d'une souche blanche de la région de Tizi Ouzou en Algérie. In: $12^{\text {es }}$ Journées de la recherche cunicole, Leman, France, 27-28 nov. 2007, 141-144

Zerrouki N., Lebas F., Berchiche M., Bolet G., 2005. Evaluation of milk production of a local Algerian rabbit population raised in the Tizi Ouzou area (Kabylia). World Rabbit Sci., 13: 39-47, doi: 10.4995/wrs.2005.530

Zerrouki N., Lebas F., Gacem M., Mefti I., Bolet G., 2014. Reproduction performances of a synthetic rabbit line and rabbits of local populations in Algeria, in two breeding locations. World Rabbit Sci., 22: 269-278, doi: 10.4995/wrs.2014.2129

\section{Summary}

Boudour K., Lankri El H., Zerrouki N.D., Aichouni A. Performances of Algerian-synthetic-strain rabbits managed with artificial insemination: Effect of the season

The objective of the study was to characterize the reproductive (fertility, reproductive productiveness, mortality) and weight performances of rabbits of the Algerian synthetic strain "ITELV 2006", conducted by artificial insemination (AI) with a semi-intensive rhythm (parturition - Al: 12-day intervals). For two consecutive years (2017 and 2018), we monitored the breeding behavior of 73 female rabbits, submitted to $327 \mathrm{Al}$, at

\section{Resumen}

Boudour K., Lankri El H., Zerrouki N.D., Aichouni A. Rendimiento de los conejos de cepa sintética argelina manejados con inseminación artificial: efecto de la estación

El objetivo del estudio fue el de caracterizar los rendimientos reproductivos (fertilidad, prolificidad, mortalidades) y ponderales de conejas de la cepa sintética argelina "ITELV2006", producidas por inseminación artificial (IA) con un ritmo semi-intensivo (intervalo de parto - IA: de 12 días). Durante dos años consecutivos (2017-2018), se llevó a cabo un seguimiento del comportamiento reproductivo de 73 conejas, objeto de 327 IA, 
the animal house of the University of Chlef (Algeria). Throughout the study period, Al was performed using semen collected from males of the same genetic type and analyzed locally. Data on $\mathrm{Al}$, palpation, parturition and weaning were recorded for all females. The season effect on weight criteria and reproductive performances of the rabbits was analyzed. All data were subjected to an analysis of variance. Females showed a good reproductive productiveness of $9.5 \pm 2.4$ total births, of which $8.4 \pm 2.9$ were born alive per parturition and $7.4 \pm 2.6$ weaned per weaning, with fertility rates of $85.6 \pm 35.1 \%$ at palpation and $69.7 \pm 45.9 \%$ at parturition. The adult weight of the females at Al was $3584 \pm 391 \mathrm{~g}$. The average weight of the rabbits was $63 \pm 13 \mathrm{~g}$ at birth and $490 \pm 81 \mathrm{~g}$ at weaning. A highly significant season effect was recorded on fertility and reproductive productiveness of rabbits, and on young rabbit weights and stillbirths, with the lowest values recorded in the hot season ( $63 \%$ fertility). However, the females remained productive during the hot season.

Keywords: Oryctolagus, rabbits, weight, fertility, conception rate, mortality, season, Algeria en las instalaciones animales de la Universidad de Chlef (Argelia). Durante toda la duración del estudio, las IA se realizaron a partir de semen recolectado de machos del mismo tipo genético y analizado localmente. Los datos relativos a la IA, a la palpación, al parto y al destete se registraron para la colectividad de las hembras. Se analizó el efecto de la estación sobre los criterios de peso y los rendimientos de reproducción de los conejos. Todos los datos fueron sometidos a un análisis de varianza. Las hembras mostraron una buena prolificidad de $9,5 \pm 2,4$ nacidos totales, de los cuáles $8,4 \pm 2,9$ nacidos vivos por parto y $7,4 \pm 2,6$ destetados por destete, con tasas de fertilidad de $85,6 \pm 35,1 \%$ a la palpación y $69,7 \pm 45,9 \%$ al parto. El peso adulto de las hembras a la IA fue de $3584 \pm 391 \mathrm{~g}$. El gazapo pesó en promedio $63 \pm 13 \mathrm{~g}$ al nacimiento y 490 $\pm 81 \mathrm{~g}$ al destete. Se registró un efecto altamente significativo de la estación sobre la fertilidad y la prolificidad de las conejas y sobre el peso y la mortinatalidad de los gazapos con los valores menores registrados durante la estación caliente $(63 \%$ de la fertilidad). Sin embargo, las hembras permanecieron productivas durante este periodo de gran calor.

Palabras clave: Oryctolagus, conejo, peso, fertilidad, prolificidad, mortalidad, estación, Argelia 